\title{
Panchromatic Sequentially Cast Ternary Polymer Solar Cells
}

\author{
Masoud Ghasemi, Long Ye, Qianqian Zhang, Liang Yan, Joo-Hyun Kim, Omar Awartani, \\ Wei You, Abay Gadisa, and Harald Ade*
}

Most recent improvements in the power conversion efficiency (PCE) of polymer solar cells (PSCs) have been attained through a better understanding of the material structure-property relationships, which have resulted in the synthesis of well-designed polymers and molecules with enhanced structural and electronic properties. ${ }^{[1-5]}$ However, most of these high-performance organic materials lack the ability to absorb a wide range of photon energies. In line with the requirement of panchromatic absorption for high current generation, low-bandgap materials, ${ }^{[4,6,7]}$ ternary blends, ${ }^{[8-10]}$ and tandem ${ }^{[11-13]}$ device structures have been employed to address the optical limitations of current state-of-the art materials. Of these, ternary PSCs that consist of two donors and one acceptor (or two acceptors and one donor) have been considered the simplest strategy to broaden the optical absorption range in PSCs, ${ }^{[14-16]}$ if the selected donors and acceptors have complementary absorption. Unfortunately, even though a few breakthroughs have been achieved, ${ }^{[8,17,18]}$ many of the ternary devices are limited by low fill factor $(\mathrm{FF})$ and/or low short-circuit current $\left(U_{\mathrm{SC}}\right)$ after adding more than $\approx 15-20 \%$ of the third component. ${ }^{[19-23]}$ Many systems with excellent optical and electronic matches often fail to deliver their promises. ${ }^{[24,25]}$ Due to the complexity of material interactions (e.g., miscibility/alloying) in ternary systems and the lack of appropriate tools to accurately study or predict these interactions, the most common practice for investigating ternary systems has largely been based on trial and error. In general, achieving favorable morphology is the limiting factor even in binary systems, ${ }^{[26-28]}$ and the complex and often unfavorable morphology of the ternary device only exacerbates the issue of morphology optimization.

Here, we utilize two donor polymers that have shown excellent photovoltaic performance in binary systems with fullerene, have ideal complementary absorption properties, but fail as a conventional ternary device due to polymer-polymer mechanical alloying on account of a negative Flory-Huggins interaction parameter $(\chi)$ between these two polymers. This is the first time that a polymer-polymer $\chi$ has been accurately measured for PSC materials. ${ }^{[25]}$ We demonstrate a unique sequential deposition strategy that circumvents these detrimental

M. Ghasemi, Dr. L. Ye, Dr. J.-H. Kim, Dr. O. Awartani,

Dr. A. Gadisa, Prof. H. Ade

Department of Physics and ORaCEL

North Carolina State University

Raleigh, NC 27695, USA

E-mail: harald_ade@ncsu.edu

Q. Zhang, Dr. L. Yan, Prof. W. You

Department of Chemistry

University of North Carolina at Chapel Hill

Chapel Hill, NC 27599, USA

DOI: 10.1002/adma.201604603 morphological outcomes associated with the traditional ternary blend. Our method gives rise to a favorable geometry and is simpler than lamination, but yields similar layered structures. While sequential casting has previously been used for the fabrication of binary PSCs, ${ }^{[29-34]}$ it has never been utilized for casting stacks of different binary bulk heterojunction $(\mathrm{BHJ})$ films, let alone for materials that form mechanical alloys. Our sequential casting approach has prevented mechanical alloying and improved device performance by deliberately selecting a difficult-to-dissolve binary $\mathrm{BHJ}$ with a crystalline donor as the bottom layer.

We studied the ternary systems of a superb, high-efficiency middle-bandgap polymer, poly(benzodithiophene-fluorinated benzotriazole) (i.e., FTAZ), ${ }^{[3,35-38]}$ and a promising and extensively studied low-bandgap polymer, poly(diketopyrrolopyrroleterthiophene) (i.e., PDPP3T). ${ }^{[39-41]}$ Although optically well matched, these two polymers in a conventional ternary blend with a fullerene molecule, [6]-phenyl $\mathrm{C}_{71}$ butyric acid methyl ester $\left(\mathrm{PC}_{71} \mathrm{BM}\right)$, only gave poor photovoltaic device performance (Figure S1, Supporting Information). This is due to mechanical alloying of the two donor polymers, as inferred from heat-flux characteristics measured by differential scanning calorimetry (DSC) as discussed below. Fortunately, we can overcome such material-induced limitations by fabricating sequentially cast ternary (SeCaT) solar cells based on these three components (FTAZ, PDPP3T, and $\mathrm{PC}_{71} \mathrm{BM}$ ). Schematics of conventional and SeCaT solar cells, energy levels, and the chemical structures of the donor polymers and the electron-acceptor molecule $\left(\mathrm{PC}_{71} \mathrm{BM}\right)$ employed in this study are shown in Figure 1a-d.

Taking advantage of the hard-to-dissolve, stable aggregation behavior of PDPP3T, we successfully fabricated SeCaT devices with a vertical phase-segregated morphology. Specifically, the semicrystalline PDPP3T:PC ${ }_{71} \mathrm{BM} \mathrm{BHJ}$ blend was first spin-cast on top of a conducting substrate from warm solution $\left(100-110^{\circ} \mathrm{C}\right)$. It provided a stable (i.e., solvent-resistant) bottom layer onto which the FTAZ:PC ${ }_{71} \mathrm{BM}$ blend is cast from roomtemperature solution without the need for an orthogonal solvent. Since holes move slightly better from PDPP3T to FTAZ than in the other direction as shown via transport measurements in bilayer, hole-only diodes (see Figure S2, Supporting Information), an inverted device architecture is used.

Current-density-voltage $(J-V)$ characteristics of the SeCaT, conventional ternary, and corresponding binary single-layer PSCs are shown in Figure $2 \mathrm{a}$, and the respective photovoltaics parameters and thicknesses are summarized in Table 1. To assure a fair and simple comparison and keeping the extracting field relatively similar by having similar overall thicknesses, the SeCaT films were fabricated in such a way that the top high-performance, medium-bandgap FTAZ layer has the same absorption coefficient as its corresponding binary 
(a)
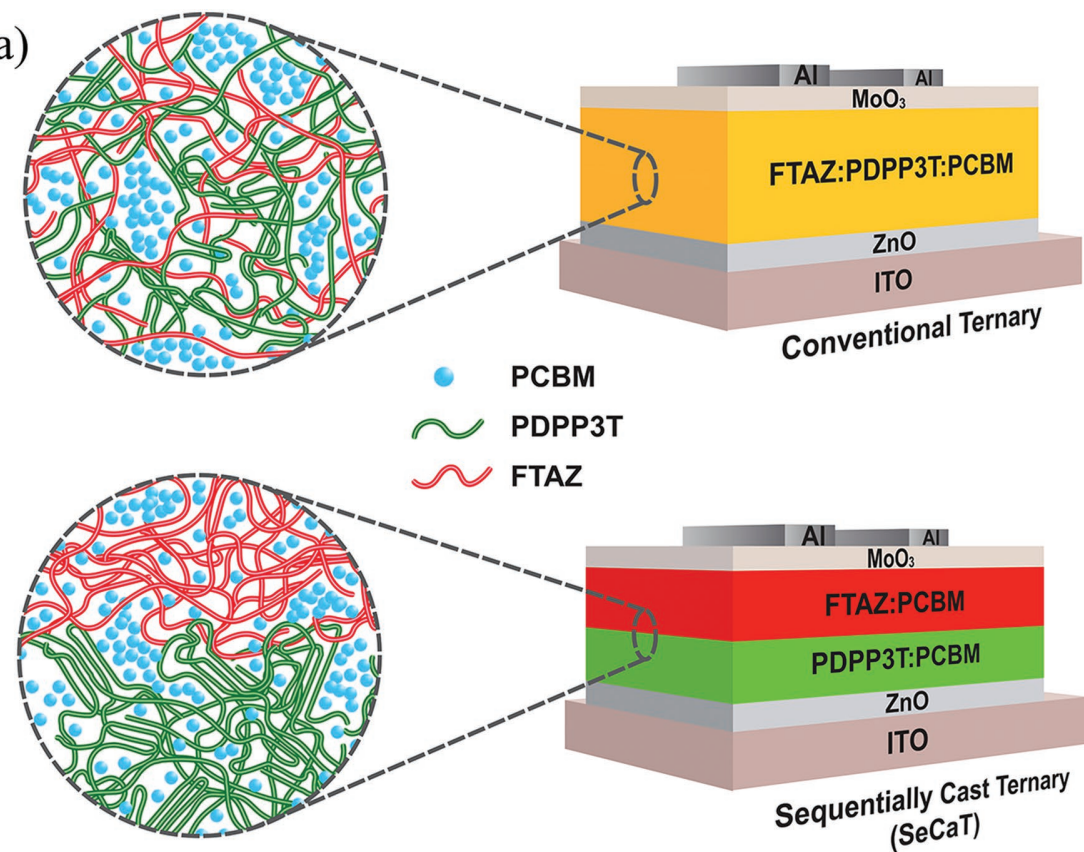

(d)

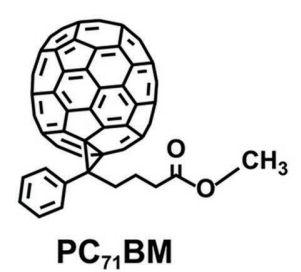

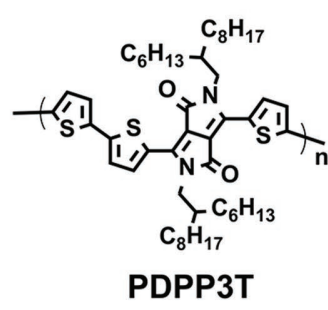

(b)

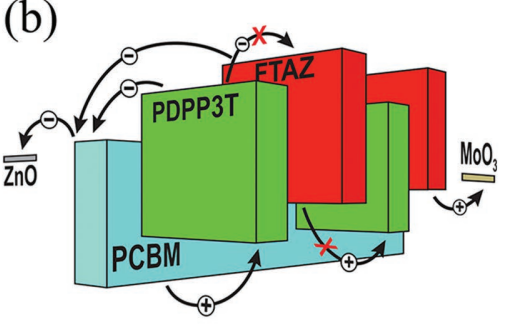

(c)
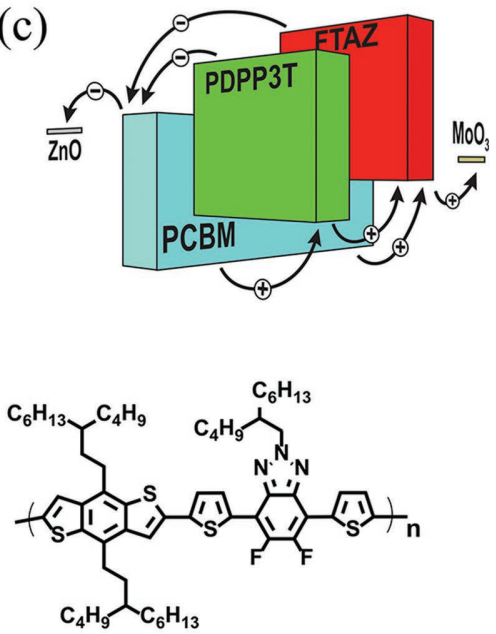

FTAZ

Figure 1. a) Schematic of conventional ternary and $\mathrm{SeCaT}$ solar cells with $\mathrm{ZnO}$ as electron and $\mathrm{MoO}_{3}$ as hole transport layers, creating an inverted device architecture. Schematic of energy levels of b) conventional ternary and c) SeCaT with vertically segregated morphology, which provides suitable pathways for electron and, more importantly, hole charge transport. d) The chemical structures of the donor polymers and the fullerene acceptor.

blend film (Figure $2 \mathrm{~b}$ ) with the thin film of PDPP3T:PC ${ }_{71} \mathrm{BM}$ added for extra absorption. Indeed, the SeCaT devices $(\mathrm{ZnO} /$ PDPP3T:PC 71 BM/FTAZ:PC 71 BM) show significantly improved performance over the FTAZ reference cell and better overall performance compared to either binary counterparts. This is primarily due to excellent contributions of both donors to the short-circuit current $\left(U_{s c}\right)$, as shown in the external quantum efficiency (EQE) spectra (Figure 2b), moderated by an opencircuit voltage $\left(V_{\mathrm{OC}}\right)$ that is in between the $V_{\mathrm{OC}}$ of the binary single-layer devices. The EQE reveals the vibronic peaks of PDPP3T and FTAZ, indicating that both donor polymers are aggregated in the binary and SeCaT devices. Furthermore, the photocurrent contribution of each polymer in SeCaT devices nearly matches the photocurrent generated in the corresponding binary-blend solar cells (Figure 2c), indicating efficient hole transfer from PDPP3T to FTAZ (for details see the Supporting Information). The EQE of the SeCaT device in the absorption region of the PDPP3T is particularly good, given that the absorbance is only $\approx 60 \%$ that of the binary.

To correlate the effect of different processing conditions with the device structures and materials interactions, resonant soft-X-ray scattering (R-SoXS) was carried out to quantitatively examine the lateral domain-size distribution within the active layer. ${ }^{[42]}$ In R-SoXS, tuning the incident photon energy makes it possible to probe the material contrast between donor-rich domains and acceptor-rich domains. The Lorentz-corrected circular averaged R-SoXS profiles of the conventional ternary, and $\mathrm{SeCaT}$ films are depicted in Figure 2c, supplemented with data from ZnO/FTAZ:PC ${ }_{71} \mathrm{BM} / \mathrm{PDPP} 3 \mathrm{~T}: \mathrm{PC}_{71} \mathrm{BM}$, and binary reference devices. The R-SoXS profiles were acquired at $284.2 \mathrm{eV}$, which is an energy below the carbon K-edge, to optimize the polymer-rich domains and the $\mathrm{PC}_{71} \mathrm{BM}$-rich domains contrast over the mass thickness contrast ${ }^{[43,44]}$ and avoid radiation damage. ${ }^{[45]}$ Since $\mathrm{PC}_{71} \mathrm{BM}$ dominates the contrast function, R-SoXS primarily maps the spatial correlations between the $\mathrm{PC}_{71} \mathrm{BM}$-rich domains even in the ternary devices. Overall, the SeCaT film with PDPP3T:PC ${ }_{71} \mathrm{BM}$ as the bottom (front) $\mathrm{BHJ}$ layer exhibits domain spacing $(28 \mathrm{~nm})$ comparable to the domain spacing of PDPP3T:PC ${ }_{71} \mathrm{BM}$ binary films $(29 \mathrm{~nm})$. This indicates that the bottom PDPP3T:PC ${ }_{71} \mathrm{BM}$ is not much disturbed by the subsequent casting of the FTAZ:PC ${ }_{71} \mathrm{BM}$. Domain spacings of conventional ternary $(39-40 \mathrm{~nm})$ and ZnO/FTAZ:PC ${ }_{71} \mathrm{BM} / \mathrm{PDPP} 3 \mathrm{~T}: \mathrm{PC}_{71} \mathrm{BM}$ SeCaT $(35 \mathrm{~nm}$ ) indicate only slightly larger phase separation in these films.

Dynamic secondary-ion mass spectroscopy (DSIMS) was further used to characterize the vertical composition gradients of the SeCaT and conventional ternary PSCs. The analysis and details of the DSIMS spectra are described in the Supporting 
(a)

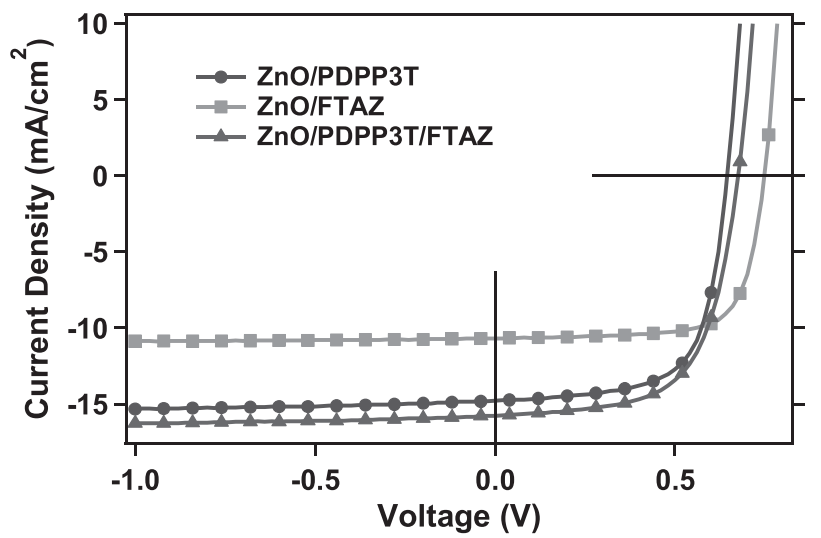

(c)

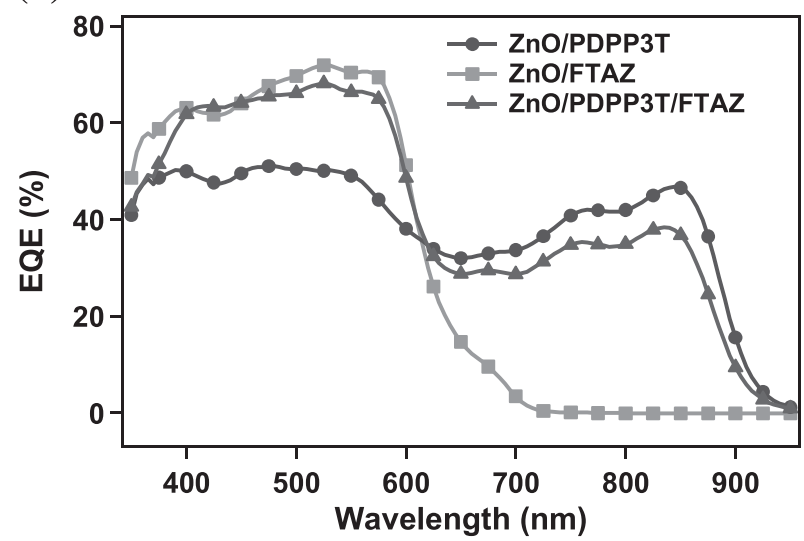

(b)

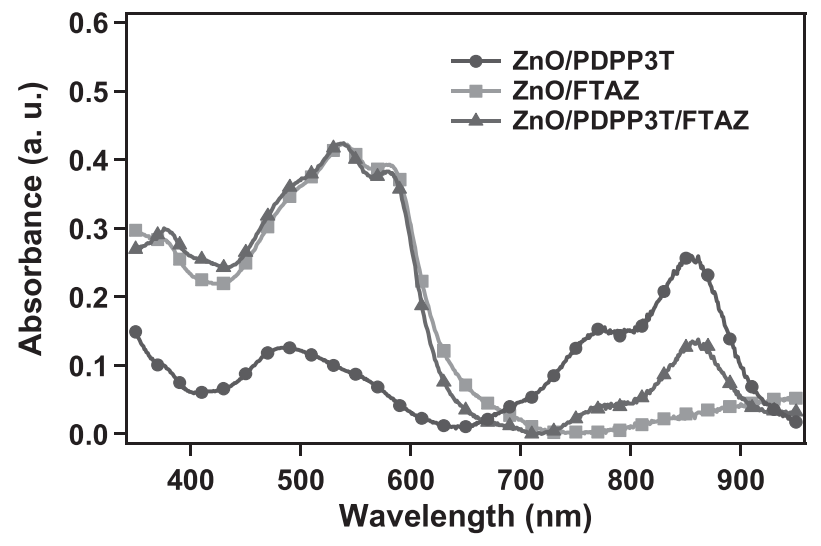

(d)

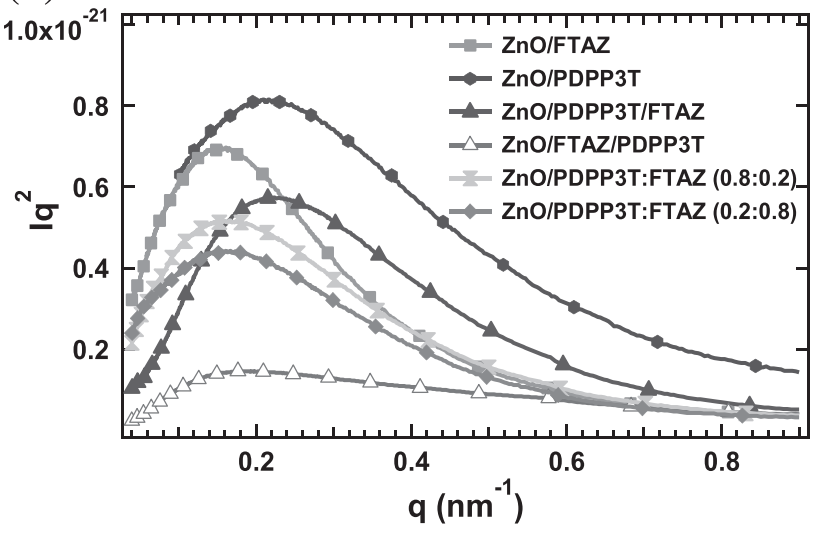

Figure 2. a) J-V curves, b) UV-vis absorption spectra of SeCaT and binary PSCs, and c) external quantum efficiency (EQE). d) Lorentz-corrected and thickness-normalized circular averaged resonant soft-X-ray scattering (R-SoXS) profiles of ZnO/FTAZ:PC $71 \mathrm{BM} \mathrm{ZnO} / \mathrm{PDPP} 3 \mathrm{~T}: \mathrm{PC} \mathrm{C}_{71} \mathrm{BM}, \mathrm{ZnO} /$

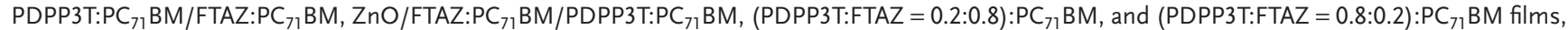
at $284.2 \mathrm{eV}$.

Information. As displayed in Figure 3a, a vertically segregated, layered structure is achieved when FTAZ:PC ${ }_{71} \mathrm{BM}$ was cast on top of the PDPP3T:PC ${ }_{71} \mathrm{BM}$ bottom layer. It is worth noting that the presence of $\mathrm{C}_{9}$ cluster ions is associated with $\mathrm{PC}_{71} \mathrm{BM}$ domains and the DSIMS results of SeCaT PSCs reveal a uniform vertical distribution of $\mathrm{PC}_{71} \mathrm{BM}$. Given that PCBM phaseseparates from either polymer, one can infer the presence of continuous charge pathway for the photogenerated electrons in the vertical direction throughout the device, which means that after exciton dissociation at the D/A interface, the electron transport can happen in a network of $\mathrm{PC}_{71} \mathrm{BM}$ domains, while holes can transfer from the PDPP3T to the FTAZ at the intermixed polymer-polymer region of the SeCaT active layer. Conventional ternary films with PDPP3T:FTAZ weight ratios of 0.2:0.8, 0.8:0.2 (Figure S5, Supporting Information), and 0.5:0.5 (Figure 3b) were also characterized by DSIMS, with

Table 1. Device performance of the binary, conventional ternary, and SeCaT PSCs.

\begin{tabular}{|c|c|c|c|c|c|c|}
\hline Device & & $\begin{array}{l}V_{O C} \\
{[\mathrm{~V}]}\end{array}$ & $\begin{array}{c}J_{\mathrm{SC}} \\
{\left[\mathrm{mA} \mathrm{cm}^{-2}\right]}\end{array}$ & $\begin{array}{l}\mathrm{FF} \\
{[\%]}\end{array}$ & $\begin{array}{c}\text { PCE avg./max. } \\
{[\%]}\end{array}$ & $\begin{array}{c}\text { Thickness } \\
{[\mathrm{nm}]}\end{array}$ \\
\hline PDPP3T:PC ${ }_{71} B M$ & Binary & 0.64 & 14.40 & 67.05 & $6.23 / 6.39$ & 80 \\
\hline FTAZ:PC ${ }_{71} B M$ & Binary & 0.75 & 10.39 & 73.82 & $5.78 / 5.86$ & 75 \\
\hline$($ PDPP3T:FTAZ $=0.2: 0.8): P C_{71} B M$ & Conventional ternary & 0.63 & 10.40 & 64.77 & $4.27 / 4.40$ & 82 \\
\hline$($ PDPP3T:FTAZ $=0.5: 0.5): \mathrm{PC}_{71} \mathrm{BM}$ & Conventional ternary & 0.63 & 11.29 & 66.73 & $4.78 / 4.81$ & 78 \\
\hline$($ PDPP3T:FTAZ $=0.8: 0.2): \mathrm{PC}_{71} \mathrm{BM}$ & Conventional ternary & 0.63 & 12.01 & 65.29 & $4.98 / 5.09$ & 90 \\
\hline PDPP3T:PC ${ }_{71}$ BM/FTAZ:PC ${ }_{71}$ BM & $\mathrm{SeCaT}$ & 0.69 & 15.67 & 61.86 & $6.64 / 6.73$ & 105 \\
\hline
\end{tabular}



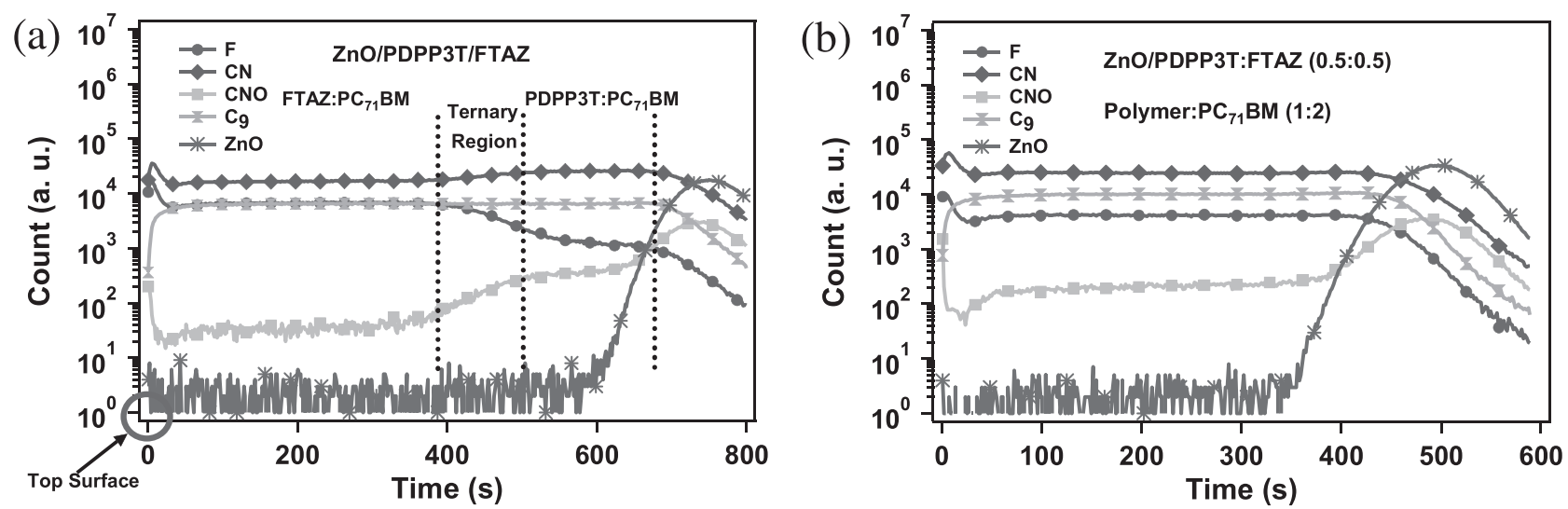

Figure 3. a,b) DSIMS depth profiles of SeCaT devices comprising PDPP3T:PC 71 BM bottom layer and FTAZ:PC $71 \mathrm{BM}$ top layer (a) and conventional ternary with 0.5:0.5 wt\% of PDPP3T:FTAZ (b), with species monitored as indicated. The top surface is at $t=0 \mathrm{~s}$.

uniform depth profiles observed for all three films. For further morphological clarification, grazing-incidence wide-angle X-ray scattering (GI-WAXS) was employed. The 2D GI-WAXS patterns and $1 \mathrm{D}$ profiles of binary, ternary blends, and SeCaT films (Figure S6, Supporting Information) reveal relatively well-defined scattering features similar to the PDPP3T:PC ${ }_{71} \mathrm{BM}$ blend, indicating higher molecular ordering of PDPP3T compared to FTAZ.

To understand the differences in the DSIMS profiles and performance, we used DSC to determine the crystallinity and polymer-polymer interaction parameter $(\chi)$. The latter provides important information associated with the miscibility of polymer-polymer systems. ${ }^{[46-48]}$ Based on the thermograms in Figure 4a, PDPP3T is a semicrystalline polymer with a melting point $\left(T_{\mathrm{m}}^{0}\right)$ of about $295^{\circ} \mathrm{C}$, which is consistent with previous reports. ${ }^{[49]}$ In contrast, FTAZ has no evidence of a melting transition and is noncrystalline. These results are consistent with the molecular ordering or respective lack thereof found in GI-WAXS. The depression of the melting-point temperature $\left(T_{\mathrm{m}}\right)$ for blended materials, using the Flory-Huggins approximation, ${ }^{[50]}$ can be used to determine the molecular interaction parameter $(\chi)$ of the polymers in the presence of a miscible diluent according to: $:^{[51]}$

$\frac{1}{T_{\mathrm{m}}}-\frac{1}{T_{\mathrm{m}}^{0}}=-\frac{R}{\Delta H_{\mathrm{f}}} \frac{v_{2}}{v_{1}} \chi\left(1-\phi_{2}\right)^{2}$

where the subscripts 1 and 2 identify the amorphous and semicrystalline polymer, respectively; $T_{\mathrm{m}}$ and $T_{\mathrm{m}}^{0}$ are the melting points of the mixture and the pure semicrystalline polymer, respectively; $R$ is the ideal gas constant; $v_{1}$ and $v_{2}$ are the molar volumes of the amorphous $\left(v_{1, \mathrm{FTAZ}}=903 \mathrm{~cm}^{3} \mathrm{~mol}^{-1}\right)$ and the semicrystalline $\left(v_{2, \text { РDРРз }}=721 \mathrm{~cm}^{3} \mathrm{~mol}^{-1}\right)$ polymer; and $\phi$ is the volume fraction. Utilizing $\chi=B v_{1} / R T$, which represents the polymer-polymer interaction that is driven by enthalpy and substitution into Equation (1), the data can be represented as shown in Figure $4 \mathrm{~b}$, and $\chi=-0.56$ can be extracted by a linear fit. We note that the excellent fit achieved indicates that $\chi$ has a negligible entropic component or a D/A ratio dependence. The critical $\chi$, i.e., $\chi_{\mathrm{c}}$, above which phase separation can occur, is generally positive. In the limit of infinite molecule weight, $\chi_{\mathrm{c}}=0$. The negative interaction parameters observed here indicates strong attractive interactions and the amorphous fractions of the two polymers form a miscible and thermodynamically stable mixture (see the Supporting Information for further details on $\chi$ calculation). $\chi$ measurements in the field are rare ${ }^{[52,53]}$ and this the first time such negative $\chi$ has been measured for semiconducting polymers. It means there is no driving force for a polymer-polymer phase separation and the polymers form a mechanical alloy in which the $\mathrm{PC}_{71} \mathrm{BM}$ is phase separated. The DSC thermograms of the second heating and cooling runs of the polymer-polymer and ternary blends represent similar melting depression and melting enthalpy trends to those that were observed in the first heating run (see Figure S8, Supporting Information).

In order to investigate the impact of mechanical alloying on charge transport, ${ }^{[54]}$ the space-charge-limited current method in a diode configuration was used to measure the hole mobility (see Figure S8, Supporting Information). For FTAZ concentrations that form an alloy, the mobility is lowered by about a factor of 2. This is consistent with the bilayer diode results that showed that hole hopping is asymmetric, with holes moving more easily from PDPP3T to FTAZ than from FTAZ to PDPP3T. Ideally, the hole from the PDPP3T hops only once along the highest occupied molecular orbital (HOMO) energy cascade (see Figure 1) to the FTAZ and a hole from the $\mathrm{PC}_{71} \mathrm{BM}$ hops once or at most twice and then remains within the FTAZ-rich phase until it reaches the electrode. Alas, in an FTAZ:PDPP3T alloy, a hole might be forced to hop back onto the lower energy HOMO of the PDPP3T, hop via a longer distance to the next FTAZ, or explore longer and more tortuous FTAZ pathways. Consequently, a miscible phase, i.e., a mechanical polymer alloy, is detrimental here for hole transport. This reduces the performance in the conventional ternary configuration of this system, which could have been ideal when only considering the matched optical and electronic properties. We suspect that transport would be even more impacted if the hopping is more asymmetric in other material pairs that have a larger HOMO offset.

To verify the existence of a mechanically alloyed phase in conventional ternary blends, the ternary films were characterized using DSC (Figure S9, Supporting Information). As shown 

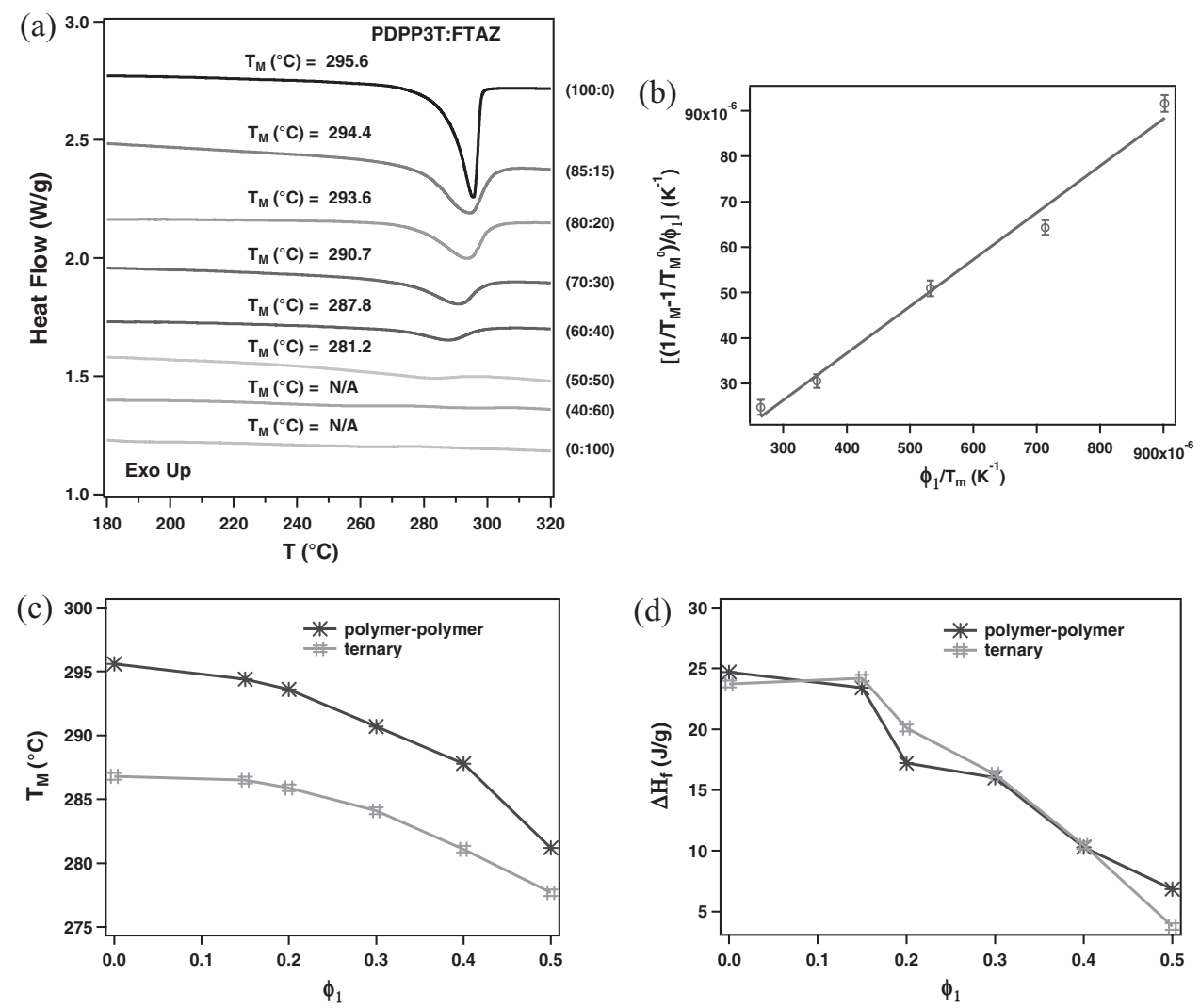

Figure 4. a) The DSC traces $\left(10^{\circ} \mathrm{C} \mathrm{min}^{-1}\right)$ of the first run of PDPP3T:FTAZ blends, b) $\left(1 / T_{\mathrm{m}}-1 / T_{\mathrm{m}}^{0}\right) / \phi_{1}$ against $\phi_{1} / T_{\mathrm{m}}$ for PDPP3T:FTAZ blends, c) melting point, and d) melting enthalpy of PDPP3T in ternary and polymer-polymer blend as a function of volume fraction of FTAZ $\left(\phi_{1}\right)$.

in Figure 4c,d, similar trends of the melting point depression and melting enthalpy of PDPP3T are reflected in the DSC thermograms. The melting-point depression offset of PDPP3T in the ternary blends compared to polymer-polymer blends is due to the additional interaction of PDPP3T with $\mathrm{PC}_{71} \mathrm{BM}$. The similar melting enthalpy of PDPP3T in polymer-polymer and ternary blends (Figure 4d) indicates that the degree of crystallinity of PDPP3T is primarily only affected by the presence of FTAZ, not by the $\mathrm{PC}_{71} \mathrm{BM}$. This indicates that PDPP3T has a much higher $\chi$ with the fullerene than with FTAZ. Furthermore, the melting-transition signature disappears in all systems as the FTAZ content reaches about $50 \%$. The similarities in melting enthalpy and melting-point depression between the polymer binary and ternary samples indicate similar and dominant polymer-polymer interactions in all blends. Such a high degree of attractive interaction between the two polymers indeed leads to unfavorable polymer-alloy morphologies that might contribute to limiting the device performance in the conventional ternary devices.

Since FTAZ is not a semicrystalline polymer, an FTAZbased bottom BHJ film is thus more unstable when casting a second layer on top in a SeCaT device. This leads to interdiffusion and mixing, which is enhanced by the negative $\chi$ during the period the film is plasticized or dissolved, resulting in DSIMS depth profiles and performance (see Figure S4 and S5, Supporting Information) that are very similar to the conventional ternary system. Open questions remain, though. For example, how much the negative $\chi$ contributes to this interdiffusion and alloying and thus poor performances is currently unclear and requires further research. Conceptually, a high $\chi$ that would be deep in the two-phase region should be significantly preferable and would lead to more stable "bilayers." How common mechanical alloying is in other ternary systems or even polymer-polymer binary systems is unclear, as this aspect has not been extensively studied previously. ${ }^{[54]}$ Our results indicate that $\chi$ measurements in general, and DSC measurements in particular, could be a useful screening tool in ternary PSC research. We note that estimates of $\chi$ from solubility parameters $\left(\chi=\alpha / k T\left(\delta_{1}-\delta_{2}\right)^{2}+0.34\right.$, where $\alpha$ is volume of one lattice segment, $\delta_{1}$ and $\delta_{2}$ are Hildebrand solubility parameters of components 1 and 2, respectively, and $k T$ is the thermal energy), ${ }^{[55]}$ would completely fail here as the method by definition only yields a positive $\chi$. Even if Hansen solubility parameters are used, a negative $\chi$ is not possible within that framework. Such failure has been previously shown to occur when strong hydrogen bonds and polar interaction are present. ${ }^{[56]}$ Our results and the likely presence of strong directional forces reinforces prior conclusions that the use of Hansen solubility parameters for PSC applications might be unreliable. ${ }^{[25,57]}$

Our results show that the bottom layer needs to be robust and retain sufficient integrity during casting of the second layer. Such a stable bottom layer can be provided by materials that are semicrystalline and are typically cast from a hot solvent. In this context, it is worth mentioning that there are a number of 
high-performing polymers that form semicrystalline films and need to be processed by warm solution $\left(\approx 100{ }^{\circ} \mathrm{C}\right)$, and some of these polymers solutions rapidly gel upon cooling to room temperature, which could make them ideal candidates for the initial layer in sequential casting. The performance of some of these materials reported in refs. [1,2,28,58] and in refs. [59-61] are listed in Table S4 in the Supporting Information. Stabilization of the initial layer to subsequent casting might also be achieved by crosslinking. ${ }^{[62-64]}$ In general, the more stable film can be either the larger- or smaller-bandgap material. If the more stable material has the higher lying HOMO, a regular device architecture is required. Conversely, for a lower HOMO, an inverted architecture is needed. This provides additional flexibility in designing SeCaT devices.

We note that ternary devices need to use more chromophores and thus more materials compared to binary devices in order to yield higher current by extending the absorption range at comparable EQE. This results in thicker devices and invariably lowers the extraction field. The lower field and larger current both increase the charge density, which causes more recombination compared the reference binary devices. We observe this intrinsic challenge here, as the FF of the SeCaT device is slightly reduced relative to the thinner binary devices. Furthermore, while in a conventional ternary device with uniform vertical morphology, the transport for both charges needs to be optimized within the same blend across the full film. In contrast, the SeCaT device can achieve the optimized charge transport rather separately in the respective layers. For example, in inverted SeCaT devices, the top/back cell can be optimized for hole transport, while the bottom/front cell can be optimized for electron transport. This relaxes the constraint on the materials systems and will allow, in principle, for additional gains and optimization once the SeCaT principle is exploited more fully.

In conclusion, we have developed a new concept in which stratified bulk-heterojunction solar-cell films were fabricated to enhance the optical spectral coverage in solar cells and have yielded improved performance (primarily from improved $J_{\mathrm{sc}}$ ) with a materials system that fails as a conventional ternary device. The method should be general, but could be particularly useful for donors with disadvantageous molecular interactions, which leads to mechanical alloying ${ }^{[51,65-67]}$ and in turn prevents the formation of a favorable phase-separated morphology in classic ternary blends. Our sequential-deposition method offers an opportunity to utilize optically matched materials systems that fail in classic ternary systems, while retaining the potential for developing truly panchromatic, scalable, and cheap organic solar cells. Moreover, the sequential-casting method results in serial pseudo-bilayer devices that function like a current-sum (three-terminal) tandem solar cell without requiring the middle electrode. Elimination of this electrode is a major advantage of our method over conventional current-sum or voltage-sum tandem cells that require an additional laborious processing step to make such an electrode that should be both transparent and sufficiently conductive or a recombination layer, respectively. Furthermore, classic voltage-sum tandem cells place enormous demands on processing control for each subcell in order to assure current matching of the two subcells. The SeCaT method not only has the potential to eliminate the demands of an electrode or recombination interlayer in tandem
PSCs but also overcomes adverse material interactions, i.e., negative $\chi$, encountered in some ternary bulk heterojunction devices, and should provide a larger processing latitude. Our results will stimulate the synthetic community to design and synthesize materials that form dissolution-resistant $\mathrm{BHJ}$ solid films, thereby allowing printing of multilayered polymer solar cells without a need for orthogonal solvents. Alternatively, development of binary systems that can be cast from orthogonal solvents is also a future possibility. Our results also indicate that $\chi$ is a material parameter that should be more widely considered when designing or synthesizing materials and understanding ternary devices. Overall, the new fabrication method and/or accompanying materials screening methodology can improve the success rate of making SeCaT and/or conventional ternary devices with improved performance.

\section{Experimental Section}

Materials: PDPP3T was purchased from Solarmer Material Inc and FTAZ was synthesized as reported previously. ${ }^{[3]} \mathrm{PC}_{71} \mathrm{BM}$ was purchased from American Dye Source Inc. The solvents used in device fabrication were purchased form Sigma-Aldrich and used as received.

Measurements: GI-WAXS and R-SoXS measurements were performed at the Advanced Light Source (ALS), Lawrence Berkeley National Laboratory. GI-WAXS and R-SoXS measurements were performed at beamline 7.3.3[68] and beamline 11.0.1.2. ${ }^{[69]}$ In the GI-WAXS measurement, a $10 \mathrm{keV} X$-ray beam was incident at a grazing angle of $0.13^{\circ}$, which maximized the scattering intensity from the bulk of the samples. In the R-SoXS measurement, samples were investigated under high vacuum $\left(1 \times 10^{-7}\right.$ Torr $)$ in order to reduce the absorption of the soft $X$-rays in air. Device characterization was carried out under AM $1.5 \mathrm{G}$ irradiation with an intensity of $100 \mathrm{~mW} \mathrm{~cm} \mathrm{~cm}^{-2}$ (Oriel Sol3A class AAA) calibrated using a National Renewable Energy Laboratory (NREL) certified standard silicon cell (KG-5 filter). Current-density-voltage curves were recorded using a Keithley 2400 digital source meter. The area of each device was $\approx 6.9 \mathrm{~mm}^{2}$ as measured using a visible-light microscope. The DSIMS was carried out using an ion time-of-flight ION TOF TOFSIMS 5 instrument, where $\mathrm{Bi}^{+}$was employed as the primary ions and $\mathrm{Cs}^{+}$was employed as the sputtering source. The film thicknesses were recorded using a KLA-Tencor P-15 profilometer. The absorbance of the films was measured using a Cary 50 UV-vis spectrophotometer. DSC samples were dissolved in chloroform at a total concentration of $20 \mathrm{mg} \mathrm{mL}^{-1}$ and stirred overnight at $50^{\circ} \mathrm{C}$. The solution was then drop cast on a glass substrate and kept under a nitrogen atmosphere for $48 \mathrm{~h}$ to remove the residual solvents. DSC measurements were carried out at a heating rate of $10^{\circ} \mathrm{C} \mathrm{min}^{-1}$ using a TA Instrument Discovery Series.

\section{Supporting Information}

Supporting Information is available from the Wiley Online Library or from the author.

\section{Acknowledgements}

The fabrication, devices measurements, $X$-ray, DSC, and DSIMS analysis, and paper preparation by North Carolina State University authors and the DSC instrument were supported by a University of North Carolina General Administration Research Opportunity Initiative (ROI) grant. The DSIMS facility at the Analytical Instrumentation Facility at North Carolina State University was supported by the State of North Carolina and the National Science Foundation (NSF). L.Y. was supported by NSF grant ECCS-1344745; Q.Z. was supported by NSFDMR-1507249 
and ONRN000141410221; and W.Y. was supported by ECCS-1344745, NSFDMR-1507249, and ONRN000141410221. X-ray data were acquired at Advanced Light Source at beamline 7.3.3 and beamline 11.0.1.2., which was supported by the Director, Office of Science, Office of Basic Energy Sciences, of the U.S. Department of Energy under Contract No. DE-AC02-05CH11231. J. Carpenter, and X. Jiao are acknowledged for assisting with the measurements and data acquisition. C. Wang, E. Schaible, and C. Zhu are acknowledged for help with X-ray experimental setup and maintenance of the beamline.

Received: August 28, 2016 Revised: October 3, 2016 Published online: November 29, 2016

[1] J. Zhao, Y. Li, G. Yang, K. Jiang, H. Lin, H. Ade, W. Ma, H. Yan, Nat. Energy 2016, 1, 15027.

[2] Y. Liu, J. Zhao, Z. Li, C. Mu, W. Ma, H. Hu, K. Jiang, H. Lin, H. Ade, H. Yan, Nat. Commun. 2014, 5, 5293.

[3] J. R. Tumbleston, B. A. Collins, L. Yang, A. C. Stuart, E. Gann, W. Ma, W. You, H. Ade, Nat. Photonics 2014, 8, 385.

[4] K. Kawashima, Y. Tamai, H. Ohkita, I. Osaka, K. Takimiya, Nat. Commun. 2015, 6, 10085.

[5] B. A. Collins, E. Gann, L. Guignard, X. He, C. R. McNeill, H. Ade, J. Phys. Chem. Lett. 2010, 1, 3160.

[6] F. Guo, N. Li, F. W. Fecher, N. Gasparini, C. O. R. Quiroz, C. Bronnbauer, Y. Hou, V. V Radmilovic, V. R. Radmilovic, E. Spiecker, K. Forberich, C. J. Brabec, Nat. Commun. 2015, 6, 7730.

[7] Z. He, B. Xiao, F. Liu, H. Wu, Y. Yang, S. Xiao, C. Wang, T. P. Russell, Y. Cao, Nat. Photonics 2015, 9, 174.

[8] Y. (M.) Yang, W. Chen, L. Dou, W.-H. Chang, H.-S. Duan, B. Bob, G. Li, Y. Yang, Nat. Photonics 2015, 9, 190.

[9] J. Zhang, Y. Zhang, J. Fang, K. Lu, Z. Wang, W. Ma, Z. Wei, J. Am. Chem. Soc. 2015, 137, 8176.

[10] S. Zhang, L. Ye, J. Hou, Adv. Energy Mater. 2016, 6, 1502529.

[11] N. Li, C. J. Brabec, Energy Environ. Sci. 2015, 8, 2902.

[12] J. You, L. Dou, K. Yoshimura, T. Kato, K. Ohya, T. Moriarty, K. Emery, C.-C. Chen, J. Gao, G. Li, Y. Yang, Nat. Commun. 2013, 4, 1446.

[13] H. Zhou, Y. Zhang, C.-K. Mai, S. D. Collins, G. C. Bazan, T.-Q. Nguyen, A. J. Heeger, Adv. Mater. 2015, 27, 1767.

[14] L. Lu, M. A. Kelly, W. You, L. Yu, Nat. Photonics 2015, 9, 491.

[15] T. Ameri, P. Khoram, J. Min, C. J. Brabec, Adv. Mater. 2013, 25, 4245.

[16] P. P. Khlyabich, B. Burkhart, B. C. Thompson, J. Am. Chem. Soc. 2011, 133, 14534.

[17] N. Gasparini, X. Jiao, T. Heumueller, D. Baran, G. J. Matt, S. Fladischer, E. Spiecker, H. Ade, C. J. Brabec, T. Ameri, Nat. Energy 2016, 1, 16118.

[18] L. Nian, K. Gao, F. Liu, Y. Kan, X. Jiang, L. Liu, Z. Xie, X. Peng, T. P. Russell, Y. Ma, Adv. Mater. 2016, 28, 8184.

[19] Q. An, F. Zhang, L. Li, J. Wang, Q. Sun, J. Zhang, W. Tang, Z. Deng, ACS Appl. Mater. Interfaces 2015, 7, 3691.

[20] S. Honda, T. Nogami, H. Ohkita, H. Benten, S. Ito, ACS Appl. Mater. Interfaces 2009, 1, 804.

[21] Y.-J. Hwang, H. Li, B. A. E. Courtright, S. Subramaniyan, S. A. Jenekhe, Adv. Mater. 2016, 28, 124.

[22] S. Liu, P. You, J. Li, J. Li, C.-S. Lee, B. S. Ong, C. Surya, F. Yan, Energy Environ. Sci. 2015, 8, 1463.

[23] L. Lu, T. Xu, W. Chen, E. S. Landry, L. Yu, Nat. Photonics 2014, 8, 716.

[24] P. P. Khlyabich, A. E. Rudenko, R. A. Street, B. C. Thompson, ACS Appl. Mater. Interfaces 2014, 6, 9913.

[25] P. P. Khlyabich, A. E. Rudenko, B. C. Thompson, Y.-L. Loo, Adv. Funct. Mater. 2015, 25, 5557.
[26] S. Mukherjee, C. M. Proctor, G. C. Bazan, T.-Q. Nguyen, H. Ade, Adv. Energy Mater. 2015, 5, 1500877.

[27] S. Mukherjee, X. Jiao, H. Ade, Adv. Energy Mater. 2016, 6, 1600699.

[28] W. Ma, G. Yang, K. Jiang, J. H. Carpenter, Y. Wu, X. Meng, T. McAfee, J. Zhao, C. Zhu, C. Wang, H. Ade, H. Yan, Adv. Energy Mater. 2015, 5, 1501400.

[29] J. C. Aguirre, S. A. Hawks, A. S. Ferreira, P. Yee, S. Subramaniyan, S. A. Jenekhe, S. H. Tolbert, B. J. Schwartz, Adv. Energy Mater. 2015, 5, 1402020.

[30] W. Chen, Z. Du, M. Xiao, J. Zhang, C. Yang, L. Han, X. Bao, R. Yang, ACS Appl. Mater. Interfaces 2015, 7, 23190.

[31] P. Cheng, J. Hou, Y. Li, X. Zhan, Adv. Energy Mater. 2014, 4, 1301349 .

[32] Y. Liu, F. Liu, H.-W. Wang, D. Nordlund, Z. Sun, S. Ferdous, T. P. Russell, ACS Appl. Mater. Interfaces 2015, 7, 653.

[33] J. J. van Franeker, S. Kouijzer, X. Lou, M. Turbiez, M. M. Wienk, R. A. J. Janssen, Adv. Energy Mater. 2015, 5, 1500464.

[34] Y. Wang, X. Zhan, Adv. Energy Mater. 2016, 6, 1600414.

[35] J. R. Tumbleston, A. C. Stuart, E. Gann, W. You, H. Ade, Adv. Funct. Mater. 2013, 23, 3463

[36] S. C. Price, A. C. Stuart, L. Yang, H. Zhou, W. You, J. Am. Chem. Soc. 2011, 133, 4625 .

[37] W. Li, L. Yang, J. R. Tumbleston, L. Yan, H. Ade, W. You, Adv. Mater. 2014, 26, 4456.

[38] W. Li, S. Albrecht, L. Yang, S. Roland, J. R. Tumbleston, T. McAfee, L. Yan, M. A. Kelly, H. Ade, D. Neher, W. You, J. Am. Chem. Soc. 2014, 136, 15566.

[39] J. C. Bijleveld, A. P. Zoombelt, S. G. J. Mathijssen, M. M. Wienk, M. Turbiez, D. M. de Leeuw, R. A. J. Janssen, J. Am. Chem. Soc. 2009, 131, 16616.

[40] W. Ma, J. R. Tumbleston, L. Ye, C. Wang, J. Hou, H. Ade, Adv. Mater. 2014, 26, 4234.

[41] L. Ye, S. Zhang, W. Ma, B. Fan, X. Guo, Y. Huang, H. Ade, J. Hou, Adv. Mater. 2012, 24, 6335.

[42] H. Ade, A. P. Hitchcock, Polymer 2008, 49, 643.

[43] S. Mukherjee, C. M. Proctor, J. R. Tumbleston, G. C. Bazan, T.-Q. Nguyen, H. Ade, Adv. Mater. 2015, 27, 1105.

[44] M. Zhang, X. Guo, W. Ma, H. Ade, J. Hou, Adv. Mater. 2015, 27, 4655.

[45] T. Coffey, S. G. Urquhart, H. Ade, J. Electron Spectrosc. Relat. Phenom. 2002, 122, 65.

[46] N. Li, F. Machui, D. Waller, M. Koppe, C. J. Brabec, Sol. Energy Mater. Sol. Cells 2011, 95, 3465

[47] F. Machui, S. Rathgeber, N. Li, T. Ameri, C. J. Brabec, J. Mater. Chem. 2012, 22, 15570.

[48] C. Müller, T. A. M. Ferenczi, M. Campoy-Quiles, J. M. Frost, D. D. C. Bradley, P. Smith, N. Stingelin-Stutzmann, J. Nelson, Adv. Mater. 2008, 20, 3510

[49] J. W. Jung, F. Liu, T. P. Russell, W. H. Jo, Energy Environ. Sci. 2013, 6, 3301.

[50] P. J. Flory, Principles of Polymer Chemistry, Cornell University Press, Ithaca, NY, USA 1953.

[51] T. Nishi, T. T. Wang, Macromolecules 1975, 8, 909

[52] F. Liu, D. Chen, C. Wang, K. Luo, W. Gu, A. L. Briseno, J. W. P. Hsu, T. P. Russell, ACS Appl. Mater. Interfaces 2014, 6, 19876.

[53] D. R. Kozub, K. Vakhshouri, L. M. Orme, C. Wang, A. Hexemer, E. D. Gomez, Macromolecules 2011, 44, 5722.

[54] Z. Wang, Y. Zhang, J. Zhang, Z. Wei, W. Ma, Adv. Energy Mater. 2016, 6, 1502456.

[55] S. Kouijzer, J. J. Michels, M. van den Berg, V. S. Gevaerts, M. Turbiez, M. M. Wienk, R. A. J. Janssen, J. Am. Chem. Soc. 2013, 135, 12057.

[56] M. Rubinstein, R. H. Colby, Polymer Physics, OUP, Oxford, UK, 2003 
[57] D. Leman, M. A. Kelly, S. Ness, S. Engmann, A. Herzing, C. Snyder, H. W. Ro, R. J. Kline, D. M. DeLongchamp, L. J. Richter, Macromolecules 2015, 48, 383.

[58] H. Lin, S. Chen, Z. Li, J. Y. L. Lai, G. Yang, T. McAfee, K. Jiang, Y. Li, Y. Liu, H. Hu, J. Zhao, W. Ma, H. Ade, H. Yan, Adv. Mater. 2015, 27, 7299.

[59] H. Choi, S.-J. Ko, T. Kim, P.-O. Morin, B. Walker, B. H. Lee, M. Leclerc, J. Y. Kim, A. J. Heeger, Adv. Mater. 2015, 27, 3318.

[60] J. A. Bartelt, J. D. Douglas, W. R. Mateker, A. El Labban, C. J. Tassone, M. F. Toney, J. M. J. Fréchet, P. M. Beaujuge, M. D. McGehee, Adv. Energy Mater. 2014, 4, 1301733.

[61] H. Hu, K. Jiang, G. Yang, J. Liu, Z. Li, H. Lin, Y. Liu, J. Zhao, J. Zhang, F. Huang, Y. Qu, W. Ma, H. Yan, J. Am. Chem. Soc. 2015, $137,14149$.

[62] G. Griffini, J. D. Douglas, C. Piliego, T. W. Holcombe, S. Turri, J. M. J. Fréchet, J. L. Mynar, Adv. Mater. 2011, 23, 1660.
[63] C.-H. Hsieh, Y.-J. Cheng, P.-J. Li, C.-H. Chen, M. Dubosc, R.-M. Liang, C.-S. Hsu, J. Am. Chem. Soc. 2010, 132, 4887.

[64] M. Jørgensen, K. Norrman, S. A. Gevorgyan, T. Tromholt, B. Andreasen, F. C. Krebs, Adv. Mater. 2012, 24, 580.

[65] Y. Nishio, T. Haratani, T. Takahashi, R. S. J. Manley, Macromolecules 1989, 22, 2547.

[66] Z. Qiu, T. Ikehara, T. Nishi, Polymer 2003, 44, 2799.

[67] E. Zhou, J. Cong, K. Hashimoto, K. Tajima, Adv. Mater. 2013, 25, 6991.

[68] A. Hexemer, W. Bras, J. Glossinger, E. Schaible, E. Gann, R. Kirian, A. MacDowell, M. Church, B. Rude, H. Padmore, J. Phys.: Conf. Ser. 2010, 247, 12007.

[69] E. Gann, A. T. Young, B. A. Collins, H. Yan, J. Nasiatka, H. A. Padmore, H. Ade, A. Hexemer, C. Wang, Rev. Sci. Instrum. 2012, 83, 045110 